\title{
Design of Ethernet-CAN Protocol Conversion Module Based on STM32
}

\author{
Yuping Zhang ${ }^{1}$, Xianghui Feng ${ }^{1}$ and Yunxiang Guo ${ }^{2}$ \\ ${ }^{1}$ School of Measurement and Control Technology and Communications Engineering \\ The Higher Educational Key Laboratory for Measuring \& Control Technology and \\ Instrumentations of Heilongjiang Province, Harbin University of Science and \\ Technology, Harbin, China \\ ${ }^{2}$ Heilongjiang Institute of Technology, Harbin, China \\ hityuping2005@163.com,fxh125@foxmail.com
}

\begin{abstract}
CAN bus is ISO international standard serial communication protocol. It is one of the most widely used fieldbus in the world. It has become the standard bus of embedded industrial control LAN. Ethernet is the most common communication protocol standard that is applied in the existing LAN. Networked industrial control usually adopts fieldbus and Ethernet network, thus the protocol conversion problems of the heterogeneous network composed of Ethernet and CAN bus has become one of the research hotspots in the technology of the industrial control network. STM32F103RC ARM microprocessor was used in the design of the Ethernet-CAN protocol conversion module, the simplified TCP/IP communication protocol - uIP protocol was adopted to improve the efficiency of the protocol conversion and guarantee the stability of the system communication. The results of the experiments show that the designed module can realize high-speed and transparent protocol conversion.
\end{abstract}

Keywords: Ethernet; CAN bus; protocol conversion; STM32; uIP

\section{Introduction}

Among the modern industry control field, bottom control systems are mostly using fieldbus technology. Most of the upper layer control and management systems consider a computer with an Ethernet interface as a terminal. In the field of industrial control, CAN (Controller Area Network) bus technology has been widely recognized and widely used in industrial automation, because of its high performance and reliability and it has been applied widely in the ship, medical equipment, intelligent building and other fields [1]. However, its communication distance is short when using high-speed communication. And the less access node shortcomings limited its wider application. Ethernet technology has the advantages of far transmission distance and easy networking, which can make up for the shortcomings of the CAN bus [2], and meanwhile it has the function of shielding the underlying differences in the control network. So this kind of CAN and Ethernet integrated heterogeneous network has been widely used. Besides, it makes the problem of two kinds of protocol conversion become the academic research hot pot. In order to solve the problems of heterogeneous network networking communication, in literature [3] it proposed a solution based on the C8051F040, and the ability of the data processing has been limited as the use of low-speed 51-core chip. Other solutions (such as described in literature [4] solution) existence the problems of the high cost, transmission opaque and so on. 
According to the low cost, high efficiency and transparent transmission requirements, the Ethernet-CAN protocol conversion scheme based on STM32 has been put forward in this paper. The conversion scheme makes it possible the advantages of Ethernet technology and CAN bus technology well performed, this also gives the new ideas of solution for problems incurred.

\section{Analysis of the Related Protocols}

The network communication protocol standards used respectively by CAN bus and Ethernet are all defined hierarchically, but there is a huge difference between the two [5]. Therefore, the theoretical basis for the design of Ethernet-CAN protocol conversion model has been provided based on two protocols be analyzed respectively.

CAN protocol only has data link layer and physical layer. The data link layer is divided into LLC (Logical Link Control sublayer) sublayer and the MAC sublayer. MAC sublayer is the core of the CAN protocol, MAC sublayer is responsible for message framing, arbitration, response, error detection and calibration. The physical layer defines the actual transmission mode of signal. The technical specification does not define the characteristics of the transceiver in physical layer, so as to optimize the transmission media and the signal level according to their application [6].

In the course of the practical development of Ethernet, four-layer model based on TCP/IP protocol stack has been most widely used [7]. This four-layer structure is the network interface layer (physical layer and data link layer), network layer, transport layer and application layer respectively [8]. TCP/IP communication protocol using this hierarchical structure, each layer needs the under layer provide services to complete its tasks. The TCP/IP protocol stack used by the model is a series of protocol set that includes the IP protocol, ICMP protocol, ARP protocol, RARP protocol, TCP protocol, UDP protocols and a series of network protocols. Among them IP and TCP protocol are especially important.

According to the characteristics of CAN protocol and TCP/IP protocol $[9,10]$, the Ethernet-CAN protocol conversion reference model has been designed based on the STM32 as shown in Figure 1.

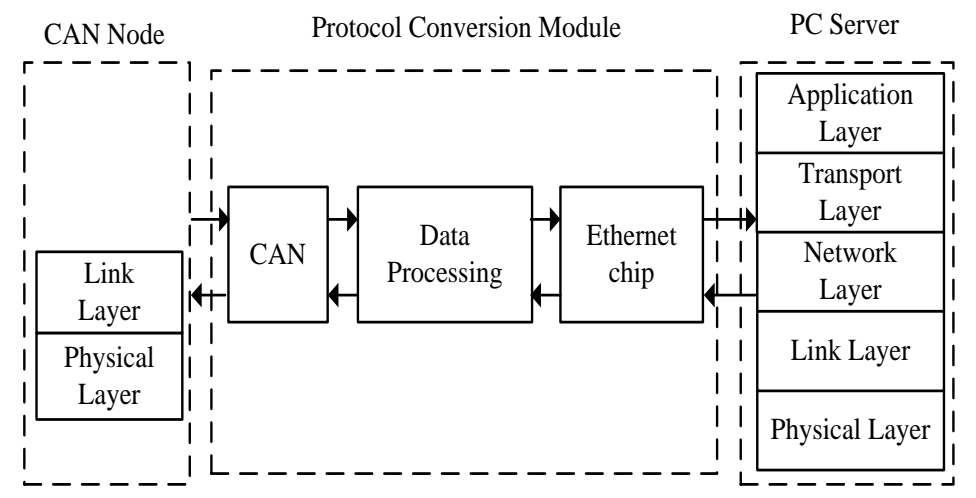

Figure 1. Protocol Conversion Model 2.

Ethernet user's data and the conversion model of CAN data frame is shown in Figure 


\begin{tabular}{|c|c|c|c|c|}
\hline \multicolumn{2}{|c|}{$\begin{array}{c}\text { Ethernet Application Layer } \\
\text { Data }\end{array}$} & \multicolumn{2}{|c|}{ User Data } & \\
\hline \multirow{3}{*}{$\begin{array}{l}\text { CAN } \\
\text { Data }\end{array}$} & Type & ID & Length & Data \\
\hline & 0xEF Standard & 4Byte & 1Byte & 0-8Byte \\
\hline & 0xFF Extended & 4Byte & 1Byte & 0-8Byte \\
\hline
\end{tabular}

Figure 2. Data Frame Transformation Model

\section{Hardware Design}

Industrial Ethernet communicates at 10/100 Mbps speed commonly, while the highest rate of CAN network is only $1 \mathrm{Mbps}$. Hardware design should take full account of the case of two networks working in different rates. In order to reduce cost and volume, the main control chip should be a cost-effective, highly integrated microcontroller. For the above two, 32-bit ARM core microcontrollers are chosen as the controller, and ENC28J60 be as Ethernet control chip [11, 12].

\subsection{CAN Controller Circuit}

STM32F103XX Enhanced Series ARM microcontrollers using high-performance Cortex$\mathrm{M} 3 / 32$ bit RISC core operate at the frequency of $72 \mathrm{MHz}$, work at $-40{ }^{\circ} \mathrm{C}$ to $+105{ }^{\circ} \mathrm{C}$ temperature range, have the built-in high-speed memory and rich peripheral resources including high-speed SPI interface and CAN controllers. Tight coupling NVIC (Nested Vectored Interrupt Controller) can achieve low latency interrupt handling and its interrupt response time is more less than 51 and ARM7 microcontroller, so its cost performance is higher. Integrated CAN controller supports CAN 2.0A and 2.0B protocol, its design goal is to handle a large number of received packets efficiently with the minimal CPU loaded. Baud rate is up to $1 \mathrm{Mbps}$, with three transmit mailboxes, two 3 level depth receiving FIFO, this kind of receiving FIFO scheme allows the CPU to handle application layer tasks for a long time without losing packets.

CAN transceiver chip produced by Texas Instruments is the $3.3 \mathrm{~V}$ high-speed CAN transceiver SN65HVD230, which has high communication speed, good anti-interference ability and high reliable of the serial communication capabilities by CAN bus. Its connection relation with the STM32 is shown in Figure 3.

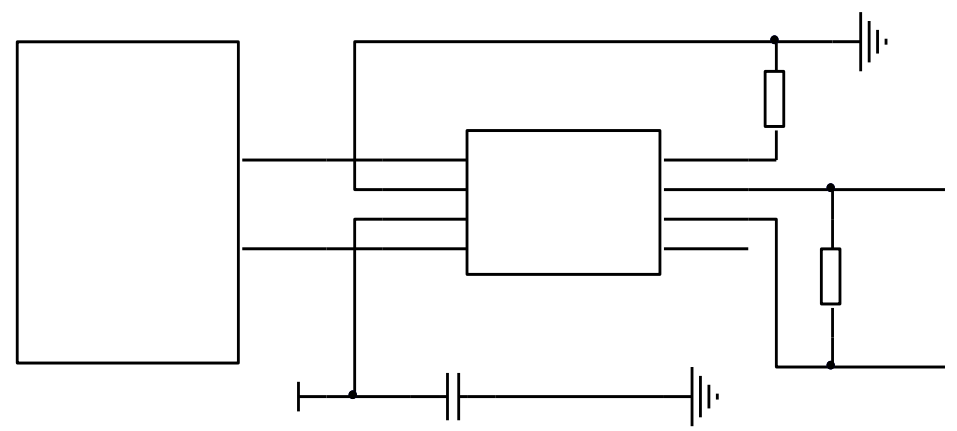

Figure 3. CAN Controller Driver Circuit 


\subsection{Ethernet Driver Circuit}

ENC28J60 is a Ethernet controller chip according with all protocol standard of IEEE 802.3. It has a standard serial peripheral interface (Serial Peripheral Interface, SPI), receives and sends data and commands by SPI transceiver, data transmission rate reached $10 \mathrm{Mb} / \mathrm{s}$. It has a $8 \mathrm{~K}$ bytes double-ended buffer which send/receive data buffer size can be configured. It also provides an internal DMA module for implementing rapid data throughput and hardware support IP checkout and calculations. The state of network activity is indicated by the LED connected with two special pins. In addition to its excellent electrical characteristics and a variety of packaging form make it be widely used in the field of Industrial Ethernet.

Using ENC28J60 as the Ethernet main chip, which connect with STM32 main controller by the SPI interface as shown in Figure 4.

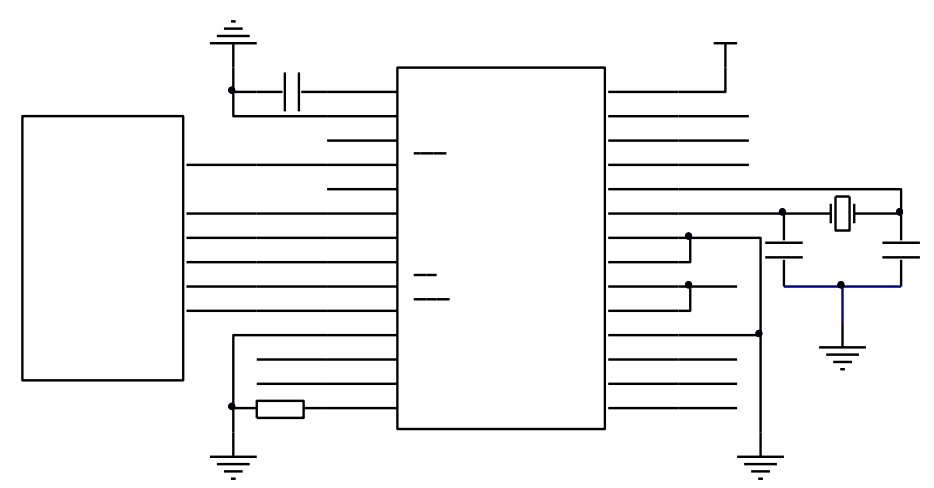

Figure 4. ENC28J60 Driver Circuit

\section{Software Design}

Software design consists of two parts, the Ethernet protocol transplant and the bidirectional communication program. In order to meet the demand for industrial Ethernet and embedded devices, Ethernet protocol should choose thin and highly reliable protocol stack, while the bidirectional communication program should ensure real-time and reliability. Therefore, the embedded TCP/IP protocol stack-uIP has been used in software design, and established a TCP connection for the data transmission.

\subsection{Ethernet Protocol Transplant}

According to the Ethernet-CAN protocol conversion model be showed in Figure 1, the software programs has been designed for implementing forwarding between Ethernet data and CAN data. The follows introduce the transplant of Ethernet protocol and Ethernet-CAN bidirectional data transceiver program.

Opening source uIP stack provides a solution for establishing TCP/IP protocol stack by embedded processors. Because of uIP protocol stack is designed for embedded systems, removing not commonly used functions in complete TCP/IP protocol stack, simplifying the communication process, but retaining the protocols network communication must use. The focus of design is IP, TCP, ICMP, UDP and ARP, these network layer and transport layer protocols, to ensure their code versatility and structural stability. The relationship between uIP protocol stack and the bottom of the system or high-level application are shown in Figure 5 . 


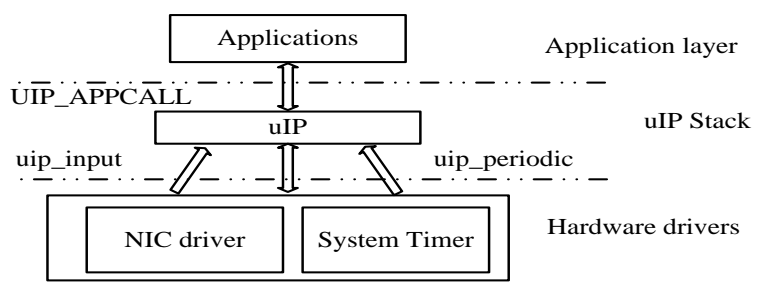

Figure 5. ulP in the System

As it can be seen from Figure 5, uIP protocol stack provides mainly two functions for the bottom of system: uip_input and uip_periodic. In addition, it contacts with application program mainly through UIP_APPCALL function. Because uIP protocol stack needs to use clock timers providing services for TCP and ARP, we need STM32 to provide a timer as clock unit, providing $10 \mathrm{~ms}$ timing. The uIP1.0 version has been selected as a portable version in this paper, ported to STM32F103RC, so as to achieve the above communication protocol.

\subsection{The Send and Receive Program of Ethernet-CAN}

Because CAN bus can only achieve half-duplex communication mode, the entire network is divided into uplink and downlink. The sending and receiving process of whole Ethernet-CAN uses interrupt mode, and transmits data by TCP protocol to ensure the data transmission is efficient and stable.

In the downlink communication, when the Ethernet receives a data packet, it puts the data into a global buffer uip_buf, the size of packet is constrained by the global variable uip_len. The data from Ethernet is parsed into the data packet of CAN network, and then send the function by calling CAN, send the grouped data packet to the CAN bus. Figure 6 shows the downlink communication.

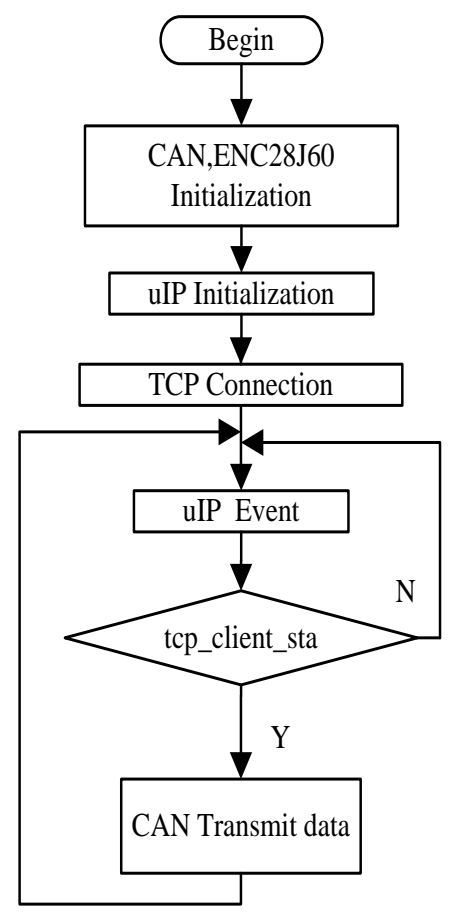

Figure 6. Downlink Communication Flow 
In the uplink communication, when the serial port of CAN receives a complete data frame, enters the CAN interrupt program and sends the data into the global buffer uip_buf. The size of buffer is assigned to uip_len, and the appropriate flag is set. When the cycle timer is triggered, calling uIP periodic function uip_periodic(), executing TCP service program, sending the packet to the network. Figure 7 shows the uplink communication.

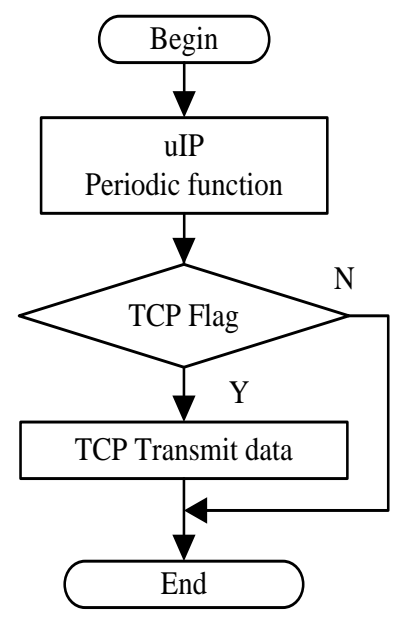

Figure 7. Uplink Communication Flow

\section{Experimental Testing and Data Analysis}

In order to provide reliable data transmission, the data is transmitted by TCP connection. Setting your computer as a server end model, using TCP Server protocol, setting the IP address to 192.168 .1 .103 , selecting the 1400 port. Ethernet-CAN conversion module is connected to the CAN network (CAN protocol is at the highest rate of $1 \mathrm{Mbps}$, sends a large number of the same data in a certain time interval). After establishing a connection, the Ethernet-CAN conversion module sends and receives data is shown in Figure 8.

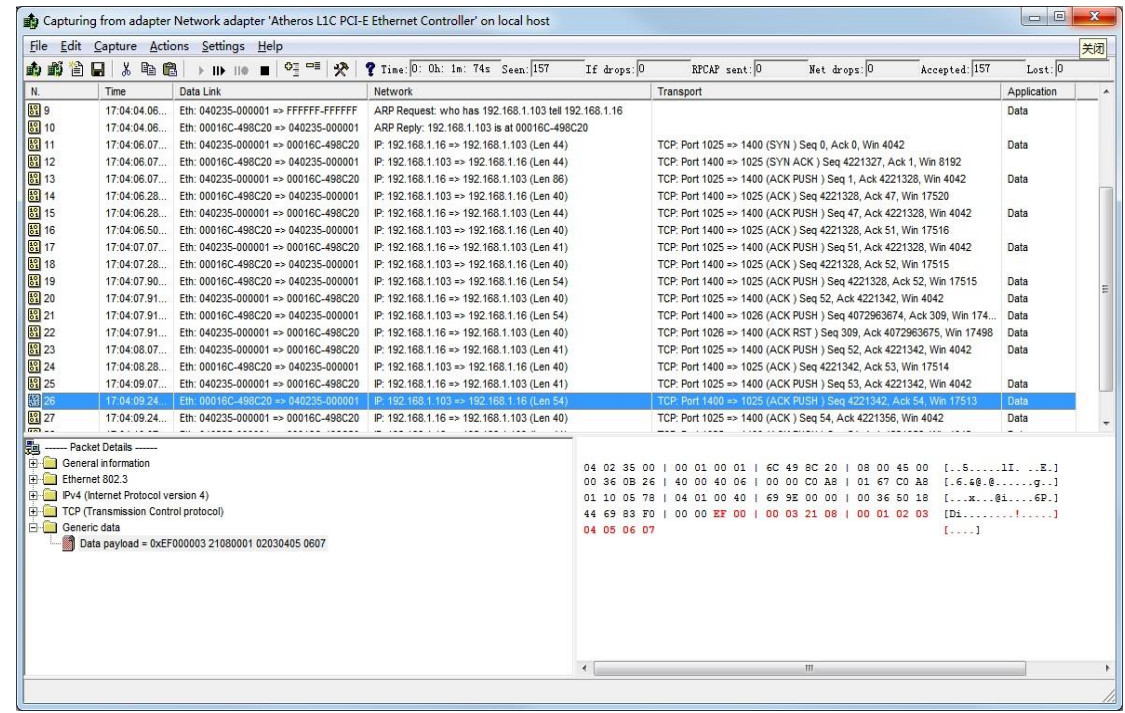

Figure 8. Send and Receive Data 
In these data, EF 00000321080001020304050607 is a frame of Ethernet user's data, EF is the type of CAN frame, 0321 is the identifier of standard frame, 00 01020304050607 is the user's data of CAN. It is verified that the system can forward user's data high-speed and steadily by the tests of sending and receiving a lots of data.

\section{Conclusion}

Ethernet-CAN protocol conversion reference model based on the STM32 is presented, according to the characteristics of the CAN protocol and the TCP/IP protocol adopted by Ethernet. Considering the case that two kinds of network work at the same time at different rates and in order to reduce the cost and the volume, high cost performance and high integration 32-bit ARM STM32F103RC is used as main control chip of microcontroller. Ethernet-CAN protocol conversion module has been designed. The compact and high reliability embedded TCP/IP protocol stack uIP is adopted and the way of TCP connection was established for data transmission. The experimental results show that the module can realize data be transmitted stably and reliably in high-speed Ethernet and low-speed CAN network.

\section{References}

[1] R. Yuntao, Z. Jijun and Z. Yongyun, "CAN Principle and application technology", M. Beijing University of Aeronautics and Astronautics Press, pp. 78-83.

[2] X. Xiren, "Computer Network (fifth edition)", M. Electronic Industry Press, (2008), pp. 121-127.

[3] C. Xing and G. Jian-zhou, "Design of Ethernet-CAN conversion interface circuit based on C8051F040", Electronic Design Engineering, vol. 19, no. 1, (2011), pp. 96-98.

[4] H. You-bao, L. Shi-yin, X. Xiao-ju and P. Li, "Design and Realization of Embedded CAN-Ethernet Access Gateway", J. Micro computer information, no. 02, (2009), pp. 32-34.

[5] W. Juan and J. Dao-xia, "Protocol Conversion Between Ethernet and CAN Bus", Computer and Modernization, no. 12, (2011), pp. 20-26.

[6] C. Hua Zeng, M. Pan and S. Yang Wang, "CAN Bus Communication System Based On SOC Technology", C. 2010 International Conference on Intelligent Computing and Integrated Systems, (2010).

[7] A. Rodriguez and J. Gatrell, TCP/IP and authoritative guide, M. Tsinghua University Press, (2002).

[8] Y.-D. Wang Dai Xiao-miao, "A Ethernet Interface Solution Based on TCP/IP Protocol”, Proceedings of 2012 IEEE 11th International Conference on Signal Processing (ICSP 2012), (2012), pp. 1521-1525.

[9] W. Sanqing, "Transplantation and Analysis of embedded network protocol of UIP based STM32", D. Wuhan University of Technology, (2011), pp. 15-26.

[10] F. Xiao and Z. Zhu, "Research and Realization of CAN-TCP/IP Embedded Gateway Based on uCLinux", C. 2010 International Conference on Computer and Communication Technologies in Agriculture Engineering (CCTAE 2010), vol. 78-80, (2010).

[11] ST Microelectronics, STM32F103XX Data Sheet, EB/OL, www.st.com, (2011).

[12] MICROCHIP, ENC28J60 Data Sheet, EB/OL. www.microchip.com, (2008). 
International Journal of Future Generation Communication and Networking Vol.7, No.1 (2014) 